\title{
Mechanical Quadrature Method and Splitting Extrapolation for Solving Dirichlet Boundary Integral Equation of Helmholtz Equation on Polygons
}

\author{
Hu Li and Yanying Ma \\ School of Mathematical Sciences, University of Electronic Science and Technology of China, Chengdu, Sichuan 611731, China \\ Correspondence should be addressed to Hu Li; lihuxiwangzhixing@163.com
}

Received 20 February 2014; Revised 20 June 2014; Accepted 22 June 2014; Published 10 July 2014

Academic Editor: Bo Yu

Copyright (c) $2014 \mathrm{H}$. Li and Y. Ma. This is an open access article distributed under the Creative Commons Attribution License, which permits unrestricted use, distribution, and reproduction in any medium, provided the original work is properly cited.

We study the numerical solution of Helmholtz equation with Dirichlet boundary condition. Based on the potential theory, the problem can be converted into a boundary integral equation. We propose the mechanical quadrature method (MQM) using specific quadrature rule to deal with weakly singular integrals. Denote by $h_{m}$ the mesh width of a curved edge $\Gamma_{m}(m=1, \ldots, d)$ of polygons. Then, the multivariate asymptotic error expansion of MQM accompanied with $O\left(h_{m}^{3}\right)$ for all mesh widths $h_{m}$ is obtained. Hence, once discrete equations with coarse meshes are solved in parallel, the higher accuracy order of numerical approximations can be at least $O\left(h_{\max }^{5}\right)$ by splitting extrapolation algorithm (SEA). A numerical example is provided to support our theoretical analysis.

\section{Introduction}

We consider Helmholtz equation with Dirichlet boundary condition:

$$
\begin{gathered}
\Delta u(x)+\alpha^{2} u(x)=0, \quad x \in \Omega, \\
u_{m}(x)=g_{m}(x), \quad x \in \Gamma_{m}\left(\Gamma=\bigcup_{m=1}^{d}\right),
\end{gathered}
$$

where $\Omega \subset \mathfrak{R}^{2}$ is a polygonal domain with the curved boundary $\Gamma=\cup_{m=1}^{d} \Gamma_{m}, d>1$, and the angels between $\Gamma_{m}$ and $\Gamma_{m+1}$ are in $(0,2 \pi]$, and the function $g_{m}(x)=\left.g(x)\right|_{\Gamma_{m}}$ is known on $\Gamma_{m}$.

By the potential theory, the solutions of (1) can be represented as a single-layer potential

$$
u(y)=\int_{\Gamma} \Phi(y, x) v(x) d s_{x}, \quad y=\left(y_{1}, y_{2}\right) \in \Omega,
$$

where $x=\left(x_{1}, x_{2}\right)$ and $\Phi(y, x)$ is the foundation solution of Helmholtz equation, which is given by

$$
\Phi(y, x)=\frac{i}{4} H_{0}^{(1)}(\alpha|x-y|) .
$$

And $H_{0}^{(1)}=J_{0}+i N_{0}$ is the Hankel functions of order zero and of the first kind, where

$$
J_{0}(z)=\sum_{n=0}^{\infty} \frac{(-1)^{n}}{(n !)^{2}}\left(\frac{z}{2}\right)^{2 n}
$$

for the Bessel function of order zero and

$$
N_{0}(z)=\frac{2}{\pi}\left(\ln \frac{z}{2}+\gamma\right) J_{0}(z)+\frac{2}{\pi} \sum_{n=1}^{\infty}\left(\sum_{m=1}^{n} \frac{1}{m}\right) \frac{(-1)^{n+1}}{(n !)^{2}}\left(\frac{z}{2}\right)^{2 n}
$$

for the Neumann function of order zero. And $\gamma=0.57721 \ldots$ is Euler constant.

In what follows, in order to analyze properties of the kernel, we decompose the kernel

$$
\Phi(x, y)=k_{1}(x, y)+k_{2}(x, y),
$$

where $k_{1}(x, y)=-1 / 2 \pi \ln |x-y|$ is logarithmic singular function and $k_{2}(x, y)=i / 4-1 / 2 \pi(\ln (\alpha / 2)+\gamma)+O(\mid x-$ $y|\ln | x-y \mid)$ is a smooth function. $v(x)$ is the solution of the following equation:

$$
g(y)=\int_{\Gamma} \Phi(y, x) v(x) d s_{x}, \quad y=\left(y_{1}, y_{2}\right) \in \Gamma .
$$


Equation (7) is weakly singular BIE system of the first kind, whose solution exists and is unique as $C_{T} \neq 1[1]$, where $C_{T}$ is the logarithmic capacity (i.e., the transfinite diameter). Once $v(x)$ is solved from (7), the function $u(y)(y \in \Omega)$ can be calculated by (2).

Galerkin and collocation methods [2,3] are used to solve (7). However, the discrete matrix is full and each element has to calculate the weakly singular integral for collocation methods or the double weakly singular integral for Galerkin methods, which implies that the work calculating discrete matrix is so large as greatly to exceed to solve the discrete equations. When the numerical methods are applied, the accuracy of numerical solutions is lower at singular points [4] and the corresponding numerical results become unreliable, because the condition numbers are very large.

In the paper, MQM is proposed to calculate weakly singular integrals by Sidi quadrature rules [5], which makes the calculation of the discrete matrix becomes very simple and straightforward without any singular integrals. The convergence theory of approximations is given by estimating eigenvalues of the discrete matrix and using Anselone's collectively compact convergent theory [6], which shows that the method retains the optimal convergence order $O\left(h_{\max }^{3}\right)$ and possesses the optimal condition number $O\left(h_{\min }^{-1}\right)$. Since MQM possesses the multivariate asymptotic expansion of errors, we can construct SEA to obtain the convergence order $O\left(h_{\max }^{5}\right)$. Once discrete equations on some coarse meshes are solved in parallel, the accuracy of numerical solutions can be greatly improved by SEA.

This paper is organized as follows. Section 2 includes the singularity analysis of the integral kernels and the solution. In Section 3, the MQM is described. In Section 4, we can obtain multiparameter asymptotic expansion of errors and SEA is described. In Section 5, a numerical example is provided to verify the theoretical results.

\section{The Integral Kernels and the Solution of Singularity Analysis}

Define boundary integral operators on $\Gamma_{m}$ :

$$
\begin{array}{r}
\left(K_{q m} v_{m}\right)(y)=-\frac{1}{2 \pi} \int_{\Gamma_{m}} \Phi(x, y) v_{m}(x) d s_{x}, \\
y \in \Gamma_{q}, \quad m, q=1, \ldots, d .
\end{array}
$$

Then (7) can be converted into a matrix operator equation

$$
K V=G
$$

where $K=\left[K_{q m}\right]_{q, m=1}^{d}, V=\left(v_{1}(x), \ldots, v_{d}(x)\right)^{T}$, and $G=$ $\left(g_{1}(y), \ldots, g_{d}(y)\right)^{T}$.

Assume that $\Gamma_{m}$ can be described by the parameter mapping:

$$
x_{m}(s)=\left(x_{1 m}(s), x_{2 m}(s)\right):[0,1] \longrightarrow \Gamma_{m}, \quad 0 \leq s \leq 1,
$$

with $\left|x_{m}^{\prime}(s)\right|=\left[\left(x_{1 m}^{\prime}(s)\right)^{2}+\left(x_{2 m}^{\prime}(s)\right)^{2}\right]^{1 / 2}>0, m=1, \ldots, d$. Using Sidi periodic transformation [7]

$$
\psi_{p}(\tau)=\frac{\vartheta_{p}(\tau)}{\vartheta_{p}(1)}:[0,1] \longrightarrow[0,1], \quad p \in N
$$

where $\vartheta_{p}(\tau)=\int_{0}^{\tau}(\sin (\pi \rho))^{p} d \rho$, then operator (8) is also converted into integral operator on $[0,1]$ :

$$
\begin{gathered}
\left(\bar{B}_{q 0} \bar{v}_{q}\right)(t)=-\frac{1}{2 \pi} \int_{0}^{1} \ln \left|2 e^{-1 / 2} \sin (\pi(t-\tau))\right| \bar{v}_{q}(\tau) d \tau, \\
\left(\bar{B}_{0 q} \bar{v}_{q}\right)(t)=-\frac{1}{2 \pi} \int_{0}^{1}\left(\ln \left|\frac{\bar{x}_{q}(t)-\bar{x}_{q}(\tau)}{2 e^{-1 / 2} \sin (\pi(t-\tau))}\right|-\frac{\pi i}{2}\right. \\
\left.+\ln \frac{\alpha}{2}+\gamma\right) \bar{v}_{q}(\tau) d \tau, \\
\left(\bar{K}_{q m} \bar{v}_{m}\right)(t)=\int_{0}^{1} \Phi\left(\bar{x}_{q}(t), \bar{x}_{m}(\tau)\right) \bar{v}_{m}(\tau) d \tau, \quad q \neq m,
\end{gathered}
$$

where $\bar{x}_{q}(t)=x_{q}\left(\psi_{p}(t)\right)$ and $\bar{v}_{m}(\tau)=$ $v_{m}\left(\psi_{p}(\tau)\right)\left|x_{m}^{\prime}\left(\psi_{p}(\tau)\right)\right| \psi_{p}^{\prime}$. Then (9) can be rewritten as

$$
\left(\bar{B}_{0}+\bar{B}_{1}+\bar{K}\right) \bar{V}=\bar{G}
$$

where $\bar{B}_{0}=\operatorname{diag}\left(\bar{B}_{10}, \ldots, \bar{B}_{d 0}\right), \bar{B}_{1}=\operatorname{diag}\left(\bar{B}_{01}, \ldots, \bar{B}_{0 d}\right), \bar{K}=$ $\left[\bar{K}_{q m}\right]_{q, m=1}^{d}, \bar{V}=\left(\bar{v}_{1}(\tau), \ldots, \bar{v}_{d}(\tau)\right)^{T}, \bar{G}=\left(\bar{G}_{1}(t), \ldots, \bar{G}_{d}(t)\right)^{T}$, and $\bar{G}_{m}(t)=g_{m}\left(\psi_{p}(t)\right)$.

Let $b_{q 0}(t, \tau), b_{0 q}(t, \tau)$, and $k_{q} m(t, \tau)$ be the kernels of the integral operators $\bar{B}_{q 0}, \bar{B}_{0 q}$, and $\bar{K}_{q m}$, respectively. Then, the following results hold.

(1) $b_{q 0}(t, \tau)$ is a logarithmic singular function on $t \in[0,1]$ and $\tau \in[0,1]$.

(2) $b_{0 q}(t, \tau)$ is a continuous function on $t \in[0,1]$ and $\tau \in$ $[0,1]$.

(3) For $\Gamma_{q} \cap \Gamma_{m}=\emptyset(|q-m| \neq 1$ or $d-1), k_{q m}(t, \tau)$ is a continuous function on $t \in[0,1]$ and $\tau \in[0,1]$.

(4) The solution $\bar{v}_{q}(t)$ is a smooth function under (11).

Lemma 1. If $\Gamma_{q} \cap \Gamma_{m} \neq \emptyset(|q-m|=1$ or $d-1)$ and $p \geq 2$, then $\bar{k}_{q m}(t, \tau)\left(=\sin ^{p}(\pi t) k_{q m}(t, \tau)\right)$ and $\left(\partial n / \partial t^{n}\right) \bar{k}(t, \tau)(n=1,2)$ are continuous on $[0,1]^{2}$.

Proof. Without loss of generality, we assume that the origin $(0,0)=\Gamma_{m} \cap \Gamma_{q}$ of coordinates is a vertex with an interior angle $\theta_{m+1}$. By (6), $k_{q m}$ can be expressed by

$$
\begin{aligned}
k_{q m}(t, \tau)= & -\frac{1}{2 \pi} \ln \left[a_{0}^{2}(t)+a_{1}(\tau)^{2}-2 a_{0}(t) a_{1}(\tau) \cos \theta_{m+1}\right] \\
& +k_{2}\left(x_{q}, x_{m}\right),
\end{aligned}
$$

where $a_{0}(t)=\left|x_{q}\left(\psi_{p}(t)\right)\right|$ and $a_{1}(\tau)=\left|x_{m}\left(\psi_{p}(\tau)\right)\right|$. We assume that $a_{0}(0)=a_{1}(0)=0$; namely, $k_{q m}$ has the 
logarithmic singularity at points $(0,0)$ and is continuously differentiable in $[0,1]^{2} \backslash(0,0)$. Consider

$$
\begin{aligned}
k_{q m}(t, \tau)= & -\frac{1}{2 \pi} \ln \left(a_{0}^{2}(t)+a_{1}(\tau)^{2}\right)-\frac{1}{2 \pi} \\
& \times \ln \left[1-\frac{2 a_{0}(t) a_{1}(\tau) \cos \theta_{m+1}}{a_{0}^{2}(t)+a_{1}(\tau)^{2}}\right]+k_{2}\left(x_{q}, x_{m}\right) .
\end{aligned}
$$

We have

$$
\left|\frac{2 a_{0}(t) a_{1}(\tau) \cos \theta_{m+1}}{a_{0}^{2}(t)+a_{1}(\tau)^{2}}\right| \leq\left|\cos \theta_{m+1}\right|<1 .
$$

So the singularity of $k_{q m}(t, \tau)$ occurs in $(1 / 2 \pi) \ln \left(a_{0}^{2}(t)+\right.$ $\left.a_{1}(\tau)^{2}\right)$. In order to remove the singularity, we define

$$
\varsigma(t, \tau)=\frac{\sin ^{p}(\pi \tau)}{\psi_{p}(1)} \ln \left(a_{0}^{2}(t)+a_{1}(\tau)^{2}\right) .
$$

Since $\psi_{p}^{\prime}$ have $p$ order zero, we have the error estimate

$$
|\varsigma(t, \tau)|=o\left(\varepsilon^{p}\right)|\ln \varepsilon| \longrightarrow 0, \quad \text { as } \varepsilon \longrightarrow 0,
$$

which shows that $\varsigma(t, \tau)$ is bounded and continuous on $\left(C^{2}[0,1]\right)^{2}$ when $\varepsilon / 2 \leq t, \tau \leq \varepsilon$ for any $\varepsilon>0$.

Similarly, we can prove $(\partial / \partial t) \varsigma(t, \tau)$ and $\left(\partial^{2} / \partial t^{2}\right) \varsigma(t, \tau)$ are continuous on $(C[0,1])^{2}$, which can be obtained by

$$
\begin{aligned}
\left|\frac{\partial}{\partial t} \varsigma(t, \tau)\right| \leq & \left|\frac{\sin ^{p}(\pi \tau)}{\psi_{p}(1)} \frac{2 a_{0}(t)\left|x_{m+1}^{\prime}\left(\psi_{p}(t)\right)\right|\left|\psi_{p}^{\prime}(t)\right|}{a_{0}^{2}(t)+a_{1}^{2}(\tau)}\right| \\
& =O\left(\varepsilon^{p}\right), \quad \forall t, \tau \in\left[\frac{\varepsilon}{2}, \varepsilon\right],
\end{aligned}
$$

and $\left(\partial^{2} / \partial t^{2}\right) \varsigma(t, \tau)=O\left(\varepsilon^{p-1}\right)$. As above, $\left(\partial^{n} / \partial t^{n}\right) \varsigma(t, \tau)(n=$ $0,1, \ldots, p)$ are continuous on $(C[0,1])^{2}$. We can prove that $\left(\partial^{n} / \partial t^{n}\right) \bar{k}_{q m}(t, \tau)(n=0,1, \ldots, p)$ are also continuous on $(C[0,1])^{2}$. The proof of Lemma 1 is completed.

\section{Mechanical Quadrature Method}

Let $h_{m}=1 / n_{m}\left(n_{m} \in N, m=1, \ldots, d\right)$ be mesh widths and let $t_{m j}=(j-1 / 2) h_{m}\left(j=1, \ldots, n_{m}\right)$ be nodes. Since an integral operator $W$ is continuous, by the trapezoidal or the midpoint rule [8], we can construct Nyström approximate operator $W^{h}$ for the integral operator $W$, defined by

$$
\left(W^{h} \bar{v}_{m}\right)(t)=h_{m} \sum_{j=1}^{n_{m}} w\left(t, t_{m j}\right) \bar{v}_{m}\left(t_{m j}\right),
$$

and the error

$$
\left(W^{h} \bar{v}_{m}\right)(t)-\left(W \bar{v}_{m}\right)(t)=O\left(h_{m}^{2 l}\right), \quad l \in N .
$$

Since $\bar{B}_{q 0}$ has the singularities on $[0,1]$, by Sidi quadrature formula [5], we get the following approximations $\bar{B}_{q 0}^{h}$ of integral operator $\bar{B}_{q 0}$ :

$$
\begin{aligned}
\left(\bar{B}_{q 0}^{h} \bar{v}_{m}\right)(t)= & -\frac{1}{2 \pi} h_{m} \sum_{j=1, t \neq t_{m j}}^{n_{m}} \ln \left|2 e^{-1 / 2} \sin \pi\left(t-t_{m j}\right)\right| \\
& \times \bar{v}_{m}\left(t_{m j}\right)-\frac{h_{m}}{2 \pi} \ln \left|2 \pi e^{-1 / 2} \frac{h_{m}}{(2 \pi)}\right| \bar{v}_{m}(t),
\end{aligned}
$$

and the error

$$
\begin{aligned}
& \left(\bar{B}_{q 0}^{h} \bar{v}_{m}\right)(t)-\left(\bar{B}_{q 0} \bar{v}_{m}\right)(t) \\
& \quad=-\frac{2}{\pi} \sum_{\mu=1}^{2 l-1} \frac{\zeta^{\prime}(-2 \mu)}{(2 \mu) !}\left[\bar{v}_{m}(t)\right]^{2 \mu} h_{m}^{2 \mu+1}+O\left(h_{m}^{2 l}\right),
\end{aligned}
$$

where $\zeta^{\prime}(t)$ is the derivative of the Riemann zeta function.

Then, we can get the approximate equation of (13):

$$
\left(\bar{B}_{0}^{h}+\bar{B}_{1}^{h}+\bar{K}^{h}\right) \bar{V}^{h}=\bar{G}^{h},
$$

where $\bar{V}^{h}=\left(\bar{V}_{1}^{h}, \ldots, \bar{V}_{d}^{h}\right), \bar{V}_{m}^{h}=\left(\bar{v}_{m}\left(t_{1}\right), \ldots, \bar{v}_{m}\left(t_{n_{m}}\right)\right), \bar{B}_{0}^{h}=$ $\operatorname{diag}\left(\bar{B}_{10}^{h}, \ldots, \bar{B}_{d 0}^{h}\right), \bar{B}_{q 0}^{h}=\left[b_{q 0}\left(t_{j}, \tau_{i}\right)\right]_{j, i=1}^{n_{q}}, \bar{B}_{1}^{h}=\operatorname{diag}\left(\bar{B}_{01}^{h}, \ldots\right.$, $\left.\bar{B}_{0 d}^{h}\right), \bar{B}_{0 q}=\left[b_{0 q}\left(t_{j}, \tau_{i}\right)\right]_{j, i}^{n_{q}}, \bar{K}^{h}=\left[\bar{K}_{q m}^{h}\right]_{q, m=1}^{d}, \bar{K}_{q m}^{h}=\left[k_{q m}\left(t_{j}\right.\right.$, $\left.\left.\tau_{i}\right)\right]_{j, i=1}^{n_{q}, n_{m}}, \bar{G}^{h}=\left(\bar{G}_{1}^{h}, \ldots, \bar{G}_{d}^{h}\right)^{T}$, and $\bar{G}_{q}^{h}=\left(g_{q}\left(t_{1}\right), \ldots, g_{q}\left(t_{n_{q}}\right)\right)$. Obviously, (24) is a system of linear equations with $n(=$ $\left.\sum_{m=1}^{d} n_{m}\right)$ unknowns. Once $\bar{V}^{h}$ is solved by (24), the solution $u(y)(y \in \Omega)$ can be computed by

$$
u^{h}(y)=\sum_{m=1}^{d} h_{m} \sum_{j=1}^{n_{m}} \Phi\left(y, x\left(t_{m j}\right)\right) \bar{v}_{m}\left(t_{m j}\right) .
$$

$\bar{B}_{q 0}^{h}$ is symmetric circular matrix and has the form of

$$
\begin{aligned}
\bar{B}_{q 0}^{h}=\operatorname{circulate}( & -\frac{h_{m}}{2 \pi} \ln \left|2 e^{-1 / 2} \frac{h_{m}}{\pi}\right|, \ldots, \\
& \left.-\frac{h_{m}}{2 \pi} \ln \left|2 e^{-1 / 2} \sin \left(\pi\left(n_{m}-1\right) h_{m}\right)\right|\right) .
\end{aligned}
$$

Lemma 2 (see [9]). (1) There exists a positive $c_{1}>0$ so that the eigenvalues $\lambda_{\beta}\left(\beta=1, \ldots, n_{m}\right)$ of $\bar{B}_{q 0}^{h}$ satisfy $c_{1}>\lambda_{\beta}>$ $1 /\left(2 \pi n_{m}\right)$. (2) The condition number of $\bar{B}_{q 0}^{h}$ is $O\left(n_{m}\right)$. (3) $\bar{B}_{q 0}$ is invertible, and $\left(\bar{B}_{q 0}^{h}\right)^{-1}$ is uniformly bounded with the spectral norm $\left\|\left(\bar{B}_{q 0}^{h}\right)^{-1}\right\|=O\left(n_{m}\right)$.

Based on Lemma 2, we immediately get the following corollary. 
Corollary 3. (1) $\bar{B}_{0}^{h}$ is invertible, and $\left(\bar{B}_{0}^{h}\right)^{-1}$ is uniformly bounded with the spectral norm $\left\|\left(\bar{B}_{0}^{h}\right)^{-1}\right\|=O\left(n_{0}\right)$. (2) The condition number of $\bar{B}_{0}^{h}$ is $O\left(n_{0}\right)$, where $n_{0}=\max _{m=1}^{d} n_{m}$.

From Corollary 3, we know that (24) is equivalent to

$$
\left(E^{h}+\left(\bar{B}_{0}^{h}\right)^{-1}\left(\bar{B}_{1}^{h}+\bar{K}^{h}\right)\right) \bar{V}^{h}=\left(\bar{B}_{0}^{h}\right)^{-1} G^{h},
$$

where $E^{h}$ denotes the unit matrix.

Now we give the following definitions to discuss the approximate convergence in (27).

Define a subspace $C_{0}[0,1]=\{v(t) \in C[0,1]: v(t) /$ $\left.\sin ^{2}(\pi t) \in C[0,1]\right\}$ of the space $C[0,1]$ with a norm $\|v\|^{*}=$ $\max _{0 \leq t \leq 1}\left|v(t) / \sin ^{2}(\pi t)\right|$. Let $S_{m}^{h} \subset C_{0}[0,1]$ be a piecewise linear function subspace with base points $\left\{t_{i}\right\}_{i=0}^{n_{m}-1}$, and let $e_{j}(t) j=0, \ldots, n_{m}-1$ be base function satisfying $e_{j}\left(t_{i}\right)=\delta_{j i}$. Define a prolongation operator $P^{h_{m}}: \mathfrak{R}^{h_{m}} \rightarrow S^{h_{m}}$ satisfying

$$
P^{h_{m}} \omega=\sum_{j=0}^{n_{m}-1} \omega_{m} e_{j}(t), \quad \forall \omega=\left(\omega_{m 1}, \ldots, \omega_{m n_{m}}\right) \in \mathfrak{R}^{n_{m}}
$$

Define a restricted operator $R^{h_{m}}: C_{0}[0,1] \rightarrow \mathfrak{R}^{n_{m}}$ satisfying

$$
\begin{array}{r}
R^{h_{m}} v_{m}=\left(v_{m}\left(t_{m 1}\right), \ldots, v_{m}\left(t_{m n_{m}}\right)\right) \in \mathfrak{R}^{n_{m}}, \\
\forall v_{m} \in C_{0}[0,1] .
\end{array}
$$

Replacing $\left(\widehat{B}_{0}^{h}\right)^{-1}\left(\widehat{B}_{1}^{h}+\widehat{K}^{h}\right)=P^{h}\left(\bar{B}_{0}^{h}\right)^{-1} R^{h}\left(\bar{B}_{1}^{h}+\bar{K}^{h}\right), \widehat{V}^{h}=$ $P^{h} \bar{V}^{h}$, and $\widehat{G}=P^{h} \bar{G}^{h}$, we construct an operator equation

$$
\left(E^{h}+\left(\widehat{B}_{0}^{h}\right)^{-1}\left(\widehat{B}_{1}^{h}+\widehat{K}^{h}\right)\right) \widehat{V}^{h}=\left(\widehat{B}_{0}^{h}\right)^{-1} \widehat{G}^{h},
$$

where $P^{h}=\operatorname{diag}\left(P_{1}, \ldots, P_{d}\right)$ and $R^{h}=\operatorname{diag}\left(R_{1}, \ldots, R_{d}\right)$. Obviously, if $\widehat{V}^{h}$ is the solution of (30), then $R^{h} \bar{V}^{h}$ must be the solution of (27); conversely, if $\bar{V}^{h}$ is the solution of (27), then $P^{h} \bar{V}^{h}$ must be the solution of (30). In order to prove the convergence of MQM, we give the following lemma.

Lemma 4 (see [9]). The operator sequence $\left\{P_{q}\left(\bar{B}_{q 0}^{h}\right)^{-1} R_{q} \bar{B}_{q 0}\right.$ : $\left.C^{2}[0,1] \rightarrow C[0,1]\right\}$ is uniformly bounded and convergent to the embedding operator $I$.

Corollary 5. Let the Nyström approximation $W^{h}$ be defined by (20). (1) For $\Gamma_{q} \cap \Gamma_{m}=\emptyset$, one has

$$
P_{m}\left(\bar{B}_{m 0}\right)^{-1} R_{m} W^{h} \stackrel{c . c}{\longrightarrow}\left(\bar{B}_{m 0}\right)^{-1} W, \quad \text { in } C[0,1] \longrightarrow C[0,1]
$$

(2) For $\Gamma_{q} \cap \Gamma_{m} \neq \emptyset$, one has

$$
\begin{array}{r}
P_{m}\left(\bar{B}_{m 0}\right)^{-1} R_{m} \widetilde{K}_{q m}^{h} \stackrel{c . c}{\longrightarrow}\left(\bar{B}_{m 0}\right)^{-1} \widetilde{K}_{q m}, \\
\text { in } C[0,1] \longrightarrow C[0,1],
\end{array}
$$

where $\widetilde{K}_{q m}^{h}$ is the Nyström approximation of integral operator $\widetilde{K}_{q m}$ with the kernel $\bar{k}_{q m}(t, \tau)=\sin ^{p}(\pi t) k_{q m}(t, \tau)$ and $\stackrel{c . c}{\longrightarrow}$ denotes the collectively compact convergence.

Theorem 6. Let $\Gamma=\cup_{m=1}^{d} \Gamma_{m}$ with $C_{T} \neq 1$ and $\Gamma_{m}(m=1, \ldots$, d) be smooth curve. The operator sequence $\left\{P^{h}\left(\bar{B}_{0}^{h}\right)^{-1} R^{h}\left(\bar{B}_{1}^{h}+\right.\right.$ $\left.\left.\bar{K}^{h}\right)\right\}$ is collectively convergent to $\left(\bar{B}_{0}\right)^{-1}\left(\bar{B}_{1}+\bar{K}\right)$ in $V=$ $\left(C_{0}[0,1]\right)^{d}$ :

$$
P^{h}\left(\bar{B}_{0}^{h}\right)^{-1} R^{h}\left(\bar{B}_{1}^{h}+\bar{K}^{h}\right) \stackrel{c . c}{\longrightarrow}\left(\bar{B}_{0}\right)^{-1}\left(\bar{B}_{1}+\bar{K}\right) .
$$

Proof. Let $\Theta=\{v:\|v\| \leq 1, v \in V\}$ be a unit ball. $H=\left\{h^{1}\right.$, $\left.h^{2}, \ldots\right\}$ is the grid step sequence, where $h^{n}=\left\{h_{1}^{(n)}, h_{2}^{(n)}, \ldots\right.$, $\left.h_{d}^{(n)}\right\}$ with $n \rightarrow \infty, \max _{1 \leq m \leq d} h_{m}^{(n)} \rightarrow 0$. We arbitrarily take a sequence $\left\{Z_{h}\right\}_{h \in H}$ in space $\Theta$, where $Z_{h}=$ $\left(Z_{h 1}, Z_{h 2}, \ldots, Z_{h d}\right)^{T}$ with $\left\|Z_{h_{m}} / \sin ^{2}(\pi t)\right\|_{\infty} \leq 1, m=1, \ldots, d$. We firstly consider the first component of $P^{h}\left(\bar{B}_{0}^{h}\right)^{-1} R^{h}\left(\bar{B}_{1}^{h}+\right.$ $\left.\bar{K}^{h}\right) Z_{h}$ :

$$
\sum_{m=1}^{d} P_{1}\left(\bar{B}_{10}^{h}\right)^{-1} R_{1}\left(\bar{B}_{01}^{h}+\bar{K}_{1 m}^{h}\right) Z_{h m}
$$

For $\Gamma_{m} \cap \Gamma_{1}=\emptyset$, by Corollary $5, P_{1}\left(\bar{B}_{10}^{h}\right)^{-1} R_{1}\left(\bar{B}_{01}^{h}+\bar{K}_{1 m}^{h}\right) \stackrel{c . c}{\longrightarrow}$ $\bar{B}_{10}^{-1}\left(\bar{B}_{01}+\bar{K}_{1 m}\right)$ in $C[0,1]$, and there exists a convergent subsequence in $\left\{P_{1}\left(\bar{B}_{10}^{h}\right)^{-1} R_{1}\left(\bar{B}_{01}^{h}+\bar{K}_{1 m}^{h}\right) Z_{h m}\right\}$. For $\Gamma_{m} \cap \Gamma_{1} \neq \emptyset$, we have

$$
\begin{aligned}
& \left\|P_{1}\left(\bar{B}_{10}^{h}\right)^{-1} R_{1} \bar{K}_{1 m}^{h} Z_{h m}\right\|_{0,0} \\
& =\left\|P_{1}\left(\bar{B}_{10}^{h}\right)^{-1} R_{1} \widetilde{K}_{1 m}^{h}\left(\frac{Z_{h m}}{\sin ^{2}(\pi t)}\right)\right\|_{0,0} \\
& \leq\left\|P_{1}\left(\bar{B}_{10}^{h}\right)^{-1} R_{1} \bar{B}_{10}\right\|_{0,2}\left\|\bar{B}_{10}^{-1} \widetilde{K}_{1 m}^{h}\right\|_{2,0}\left\|\frac{Z_{h_{m}}}{\sin ^{2}(\pi t)}\right\|_{\infty} .
\end{aligned}
$$

By Lemma 4, there exists a convergent subsequence in $\left\{P_{1}\left(\bar{B}_{10}^{h}\right)^{-1} R_{1}\left(\bar{B}_{01}^{h}+\bar{K}_{1 m}^{h}\right) Z_{h m}\right\}$. Based on the above two cases it is proved that there exists an infinite subsequence $H^{(1)} \subset H$ such that the first component converges. Similarly, it can be concluded that there exists an infinite subsequence $H^{(d)} \subset$ $H^{(d-1)} \subset \cdots \subset H^{(1)} \subset H$ such that $P^{h}\left(\bar{B}_{0}^{h}\right)^{-1} R^{h}\left(\bar{B}_{1}^{h}+\bar{K}^{h}\right) Z_{h}$ converges. Therefore, $P^{h}\left(\bar{B}_{0}^{h}\right)^{-1} R^{h}\left(\bar{B}_{1}^{h}+\bar{K}^{h}\right)$ is collectively compact convergent sequence, and $P^{h}\left(\bar{B}_{0}^{h}\right)^{-1} R^{h}\left(\bar{B}_{1}^{h}+\bar{K}^{h}\right) \stackrel{P}{\rightarrow}$ $\left(\bar{B}_{0}\right)^{-1}\left(\bar{B}_{1}+\bar{K}\right)$, where $\stackrel{P}{\rightarrow}$ shows the point convergence. We complete the proof.

For the stability of MQM, we have the following corollary.

Corollary 7. Let $\Gamma=\cup_{m=1}^{d} \Gamma_{m}$ with $C_{\Gamma} \neq 1$, let $\Gamma_{m}(m=$ $1, \ldots, d)$ be smooth curve, let $\bar{B}_{0}^{h}, \bar{B}_{1}^{h}$, and $\bar{K}^{h}$ be the discrete matrices defined by (20) and (22), respectively, and let $\lambda_{i}(i=$ 
$1, \ldots, n)$ be the eigenvalues of discrete matrix $M^{h}=\bar{B}_{0}^{h}+\bar{B}_{1}^{h}+$ $\bar{K}^{h}$. Then, there exists the bound of condition number

$$
\text { Cond }\left(M^{h}\right)=\frac{\max _{1 \leq i \leq n}\left|\lambda_{i}\left(M^{h}\right)\right|}{\max _{1 \leq i \leq n}\left|\lambda_{i}\left(M^{h}\right)\right|}=O\left(h_{\text {min }}^{-1}\right),
$$

where $h_{\min }=\min _{m=1}^{d} h_{m}, h_{m}=1 / n_{m}$, is the mesh step size of $a$ curved edge $\Gamma_{m}$.

\section{Multiparameter Asymptotic Expansion of Errors and SEA}

In this section, we derive the multivariate asymptotic expansion of solution errors and describe SEA. We first provide the main result.

Theorem 8. Let $\Gamma=\cup_{m=1}^{d} \Gamma_{m}$ with $C_{\Gamma} \neq 1$. There exists a vector function $\Phi=\left(\phi_{1}, \ldots, \phi_{d}\right)^{T}$ independent of $h=\left(h_{1}, \ldots, h_{d}\right)$ such that the following multiparameter asymptotic expansion holds at nodes:

$$
\bar{V}^{h}-\bar{V}=\operatorname{diag}\left(h_{1}^{3}, \ldots, h_{d}^{d}\right) \Phi+o\left(h_{0}^{3}\right), \quad h_{0}=\max _{1 \leq m \leq d} h_{m},
$$

where $\bar{V}^{h} \in S^{d}, \bar{V} \in C^{3}[0,1]$.

Proof. By (20) and (22), there exists the asymptotic expansion

$$
\left(\bar{G}^{h}-\bar{G}\right)=\operatorname{diag}\left(h_{1}^{3}, \ldots, h_{d}^{d}\right) P^{h} R^{h} \omega+o\left(h_{0}^{3}\right),
$$

where $h=\max _{m=1}^{d} h_{m}$ and $\omega=\left(\omega_{1}, \ldots, \omega\right)^{T}$ with $\omega_{m}=$ $-\zeta^{\prime}(-2)\left(G_{m}(t)\right)^{\prime \prime} / \pi$.

Using (13), (21), (23), and (24), we obtain

$$
\begin{aligned}
\left(\bar{B}_{0}^{h}+\bar{B}_{1}^{h}+\bar{K}^{h}\right) R^{h}\left(\bar{V}^{h}-\bar{V}\right) & \\
= & \bar{V}^{h}-P^{h}\left(\bar{B}_{0}^{h}+\bar{B}_{1}^{h}+\bar{K}^{h}\right) R^{h} \bar{V} \\
= & \bar{V}^{h}-\left[\left(\bar{B}_{0}+\bar{B}_{1}+\bar{K}\right) R^{h} \bar{V}\right. \\
& \left.\quad-\operatorname{diag}\left(h_{1}^{3}, \ldots, h_{d}^{d}\right) P^{h} R^{h} \gamma\right]+o\left(h_{0}^{3}\right) \\
= & \left(\bar{G}^{h}-\bar{G}\right)+\operatorname{diag}\left(h_{1}^{3}, \ldots, h_{d}^{d}\right) P^{h} R^{h} \gamma+o\left(h_{0}^{3}\right) \\
= & \operatorname{diag}\left(h_{1}^{3}, \ldots, h_{d}^{d}\right) P^{h} R^{h} \eta+o\left(h_{0}^{3}\right),
\end{aligned}
$$

where $\gamma=\left(\gamma_{1}, \ldots, \gamma_{d}\right)^{T}$ with $\gamma_{m}=-\zeta^{\prime}(-2)\left(x^{\prime}(t) \psi^{\prime}(t) v(t)\right)^{\prime \prime} / \pi$ and $\eta=\left(\eta_{1}, \ldots, \eta_{d}\right)^{T}$ with $\eta_{m}=\omega_{m}+\gamma_{m}$. From Corollary 3 , we can obtain

$$
\begin{aligned}
& \left(E+P^{h}\left(\bar{B}_{0}^{h}\right)^{-1} R^{h}\left(\bar{B}_{1}^{h}+\bar{K}^{h}\right)\right)\left(\bar{V}^{h}-\bar{V}\right) \\
& =\operatorname{diag}\left(h_{1}^{3}, \ldots, h_{d}^{d}\right)\left(\bar{B}_{0}^{h}\right)^{-1} P^{h} R^{h} \eta+o\left(h_{0}^{3}\right) .
\end{aligned}
$$

Constructing the auxiliary equation

$$
\left(E+\left(\bar{B}_{0}\right)^{-1}\left(\bar{B}_{1}+\bar{K}\right)\right) \Phi=\left(\bar{B}_{0}\right)^{-1} \eta
$$

and its approximate equation

$$
\left(E+P^{h}\left(\bar{B}_{0}^{h}\right)^{-1} R^{h}\left(\bar{B}_{1}^{h}+\bar{K}^{h}\right)\right) \Phi^{h}=\left(\bar{B}_{0}^{h}\right)^{-1} P^{h} R^{h} \eta
$$

and substituting (42) into (40), we obtain

$$
\begin{aligned}
(E+ & \left.P^{h}\left(\bar{B}_{0}^{h}\right)^{-1} R^{h}\left(\bar{B}_{1}^{h}+\bar{K}^{h}\right)\right) \\
& \times\left(\bar{V}^{h}-\bar{V}-\operatorname{diag}\left(h_{1}^{3}, \ldots, h_{d}^{d}\right) \Phi^{h}\right)=o\left(h_{0}^{3}\right) .
\end{aligned}
$$

Since $\left(E+P^{h}\left(\bar{B}_{0}^{h}\right)^{-1} R^{h}\left(\bar{B}_{1}^{h}+\bar{K}^{h}\right)\right)$ is uniformly bounded from Theorem 6, we get

$$
\bar{V}^{h}-\overline{\mathrm{V}}-\operatorname{diag}\left(h_{1}^{3}, \ldots, h_{d}^{d}\right) \Phi^{h}=o\left(h_{0}^{3}\right) .
$$

Replacing $\Phi^{h}$ with $\Phi$, we can complete the proof.

The multiparameter asymptotic expansion (37) means that SEA can be applied to solve (7); that is, higher order accuracy $o\left(h_{0}^{3}\right)$ at coarse grid points can be obtained by solving some discrete equations in parallel. The process of SEA is as follows [10].

Step 1. Take $h^{(0)}=\left(h_{1}^{(0)}, \ldots, h_{d}^{(0)}\right)$ and $h^{(m)}=$ $\left(h_{1}^{(0)}, \ldots, h_{m}^{(0)} / 2, \ldots, h_{d}^{(0)}\right)$, and solve (24) under mesh parameters $h^{(m)}$ in parallel to get the numerical solutions $\bar{V}^{h^{(0)}}\left(t_{m j}\right)$ and $\bar{V}^{h^{(m)}}\left(t_{m j}\right), m=1, \ldots, d, j=1, \ldots, n_{m}$.

Step 2. Compute $u^{h^{(0)}}(y)$ and $u^{h^{(m)}}(y)(y \in \Omega)$, by (25), $\bar{V}^{h^{(0)}}\left(t_{m j}\right)$, and $\bar{V}^{h^{(m)}}\left(t_{m j}\right)$.

Step 3. Compute the extrapolation on the coarse grids as follows:

$$
u^{*}(y)=\frac{8}{7}\left[\sum_{m=1}^{d} u^{h^{(m)}}(y)-\left(d-\frac{7}{8}\right) u^{h^{(0)}}(y)\right] .
$$

Step 4. Compute a posteriori error estimate on the coarse grids as follows:

$$
\begin{aligned}
\mid u(y) & -\frac{1}{d} \sum_{m=1}^{d} u^{h^{(m)}}(y) \mid \\
\leq & \left|u(y)-\left[\sum_{m=1}^{d} u^{h^{(m)}}(y)-\left(d-\frac{7}{8}\right) u^{h^{(0)}}(y)\right]\right| \\
& +\left(\frac{8}{7} d-1\right)\left|\frac{1}{d} \sum_{m=1}^{d} u^{h^{(m)}}(y)-u^{h^{(0)}}(y)\right| \\
\leq & \left(\frac{8}{7} d-1\right)\left|\frac{1}{d} \sum_{m=1}^{d} u^{h^{(m)}}(y)-u^{h^{(0)}}(y)\right|+o\left(h_{0}^{3}\right) .
\end{aligned}
$$

In the actual calculation process, a posteriori error estimate is immediately used to verify the calculation accuracy. 
TABLE 1: The numerical results.

\begin{tabular}{lcccc}
\hline$\left(n_{1}, n_{2}, n_{3}, n_{4}\right)$ & $\operatorname{Re}\left(e_{A}\right)$ & $\operatorname{Im}\left(e_{A}\right)$ & $\operatorname{Re}\left(e_{B}\right)$ & $\operatorname{Im}\left(e_{B}\right)$ \\
\hline$(8,8,8,8)$ & $3.943 e-4$ & $2.459 e-4$ & $6.255 e-4$ & $2.832 e-4$ \\
$(16,8,8,8)$ & $4.730 e-4$ & $2.351 e-4$ & $6.756 e-4$ & $3.007 e-4$ \\
$(8,16,8,8)$ & $1.400 e-4$ & $1.472 e-4$ & $3.007 e-4$ & $1.688 e-4$ \\
$(8,8,16,8)$ & $1.400 e-4$ & $1.472 e-4$ & $6.756 e-4$ & $1.688 e-4$ \\
$(8,8,8,16)$ & $4.730 e-4$ & $2.351 e-4$ & $4.902 e-4$ & $2.701 e-4$ \\
Post-error & $3.134 e-4$ & $1.953 e-4$ & $2.080 e-6$ & $8.284 e-6$ \\
SEA-error & $6.841 e-6$ & $4.125 e-6$ & $7.934 e-5$ & $2.973 e-5$ \\
$(16,16,16,16)$ & $4.534 e-5$ & $2.849 e-5$ & $8.549 e-5$ & $2.788 e-5$ \\
$(32,16,16,16)$ & $5.438 e-5$ & $2.724 e-5$ & $3.849 e-5$ & $1.859 e-5$ \\
$(16,32,16,16)$ & $1.648 e-5$ & $1.729 e-5$ & $3.849 e-5$ & $1.859 e-5$ \\
$(16,16,32,16)$ & $1.648 e-5$ & $1.729 e-5$ & $8.549 e-5$ & $2.788 e-5$ \\
$(16,16,16,32)$ & $5.438 e-5$ & $2.724 e-5$ & $1.900 e-5$ & $4.377 e-5$ \\
Post-error & $3.541 e-5$ & $2.225 e-5$ & $1.398 e-8$ & $1.867 e-8$ \\
SEA-error & $1.275 e-8$ & $1.442 e-8$ & & \\
\hline
\end{tabular}

TABLE 2: The condition number.

\begin{tabular}{lccc}
\hline$\left(n_{1}, n_{2}, n_{3}, n_{4}\right)$ & $\left(2^{3}, 2^{3}, 2^{3}, 2^{3}\right)$ & $\left(2^{4}, 2^{4}, 2^{4}, 2^{4}\right)$ & $\left(2^{5}, 2^{5}, 2^{5}, 2^{5}\right)$ \\
\hline$\left|\lambda_{\min }\right|$ & $2.214 e-3$ & $1.059 e-3$ & $5.236 e-4$ \\
$\left|\lambda_{\max }\right|$ & 0.6408 & 0.6405 & 0.6404 \\
Cond & $2.894 e+002$ & $6.047 e+002$ & $1.223 e+003$ \\
\hline
\end{tabular}

\section{Numerical Example}

In this section, we carry out a numerical example for the Helmholtz equation by MQM and SEA, in order to verify the error and stability analysis in the previous sections. Let $\operatorname{Re}\left(e_{p}\right)$ be the real part of $e_{P}=\left|u^{h}(P)-u(P)\right|$ and let $\operatorname{Im}\left(e_{P}\right)$ be the imaginary part of $e_{P}=\left|u^{h}(P)-u(P)\right|$, where $P$ denotes a point. Post-error and SEA-error denote the a posteriori error and the error after SEA once, respectively.

Example 1. Consider Helmholtz equation with $\alpha=\sqrt{2}$ on a plate domain $\Omega$. We describe the boundary $\Gamma=\cup_{m}^{4} \Gamma_{m}$ with $\Gamma_{1}=\left\{\left(x_{1}, x_{2}\right) \mid 0 \leq x_{1} \leq 1, x_{2}=0\right\}, \Gamma_{2}=\left\{\left(x_{1}, x_{2}\right) \mid 0 \leq\right.$ $\left.x_{2} \leq 1, x_{1}=1\right\}, \Gamma_{3}=\left\{\left(x_{1}, x_{2}\right) \mid 0 \leq x_{1} \leq 1, x_{2}=1\right\}$, and $\Gamma_{4}=\left\{\left(x_{1}, x_{2}\right) \mid 0 \leq x_{2} \leq 1, x_{1}=0\right\}$. Dirichlet boundary conditions corresponding to the analytical solution $e^{i \times\left(x_{1}+x_{2}\right)}$ were applied to the boundary. We compute the numerical solution $u^{h}$ with $P=A(0.5,0.5)$ and $P=B(0.6,0.6)$ using $\varphi_{3}(t)$. The numerical results are listed in Tables 1 and 2 . From Table 1, we can know that the convergence rates of $u^{h}$ are $O\left(h_{\max }^{3}\right)$ for MQM and that the convergence rates of $u^{h}$ are at least $O\left(h_{\max }^{5}\right)$ for SEA. From Table 2, we can see Cond $\left.\right|_{\left(2^{k+1}, 2^{k+1}, 2^{k+1}, 2^{k+1}\right)} /$ Cond $\left.\right|_{\left(2^{k}, 2^{k}, 2^{k}, 2^{k}\right)} \approx 2(k=3,4,5)$ to indicate Corollary 7 . It verifies the stability of convergent theory for MQM. Those results coincide with the theoretical analysis made.

\section{Concluding Remarks}

To close this paper, let us make a few concluding remarks.
(1) Evaluation on entries of discrete matrices is very simple and straightforward without any singular integrals by MQM.

(2) The numerical experiments show that MQM retains the optimal convergence order $O\left(h_{\max }^{3}\right)$ and possesses the optimal condition number $O\left(h_{\text {min }}^{-1}\right)$ which shows MQM owns the excellent stability. The approximate solutions accuracy order is at least $O\left(h_{\max }^{5}\right)$ after splitting extrapolation once, which is a great improvement in accuracy.

\section{Conflict of Interests}

The authors declare that there is no conflict of interests regarding the publication of this paper.

\section{References}

[1] Y. Yan and I. H. Sloan, "On integral equations of the first kind with logarithmic kernels," Journal of Integral Equations and Applications, vol. 1, no. 4, pp. 549-579, 1988.

[2] I. H. Sloan and A. Spence, "The Galerkin method for integral equations of the first kind with logarithmic kernel: theory," IMA Journal of Numerical Analysis, vol. 8, no. 1, pp. 105-122, 1988.

[3] Y. Yi, "The collocation method for first-kind boundary integral equations on polygonal regions," Mathematics of Computation, vol. 54, no. 189, pp. 139-154, 1990.

[4] Z. C. Li, T. T. Lu, H. Y. Hu, and H. D. Cheng, "Particular solutions of Laplace's equations on polygons and new models involving mild singularities," Engineering Analysis with Boundary Elements, vol. 29, no. 1, pp. 59-75, 2005.

[5] A. Sidi and M. Israeli, "Quadrature methods for periodic singular and weakly singular fredholm integral equations," Journal of Scientific Computing, vol. 3, no. 2, pp. 201-231, 1988.

[6] P. M. Anselone, Collectively Compact Operator Approximation Theory, Prentice-Hall, Englewood Cliffs, NJ, USA, 1971.

[7] A. Sidi, "A new variable transformation for numerical integration," in Numerical Integration IV, pp. 359-374, Birkhaurer, Frankfurt, Germany, 1993. 
[8] P. Davis, Methods of Numerical Integration, Academic Press, New York, NY, USA, 2nd edition, 1984.

[9] J. Huang and T. Lu, "Splitting extrapolations for solving boundary integral equations of linear elasticity Dirichlet problems on polygons by mechanical quadrature methods," Journal of Computational Mathematics, vol. 24, no. 1, pp. 9-18, 2006.

[10] Q. Lin and T. Lu, "Splitting extrapolation for multidimensional problems," Journal of Computational Mathematics, vol. 1, pp. 4551, 1983. 


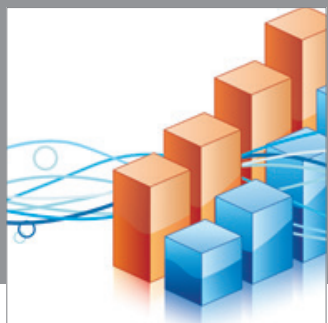

Advances in

Operations Research

mansans

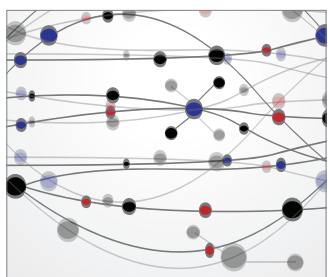

The Scientific World Journal
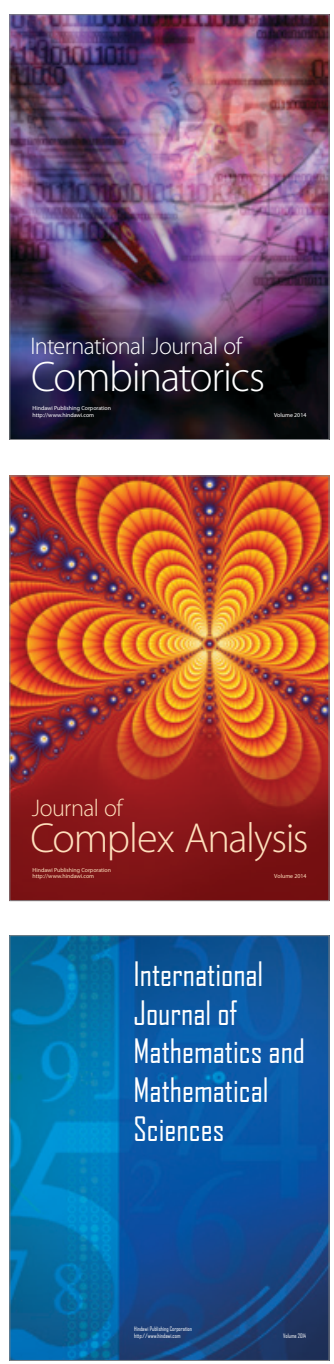
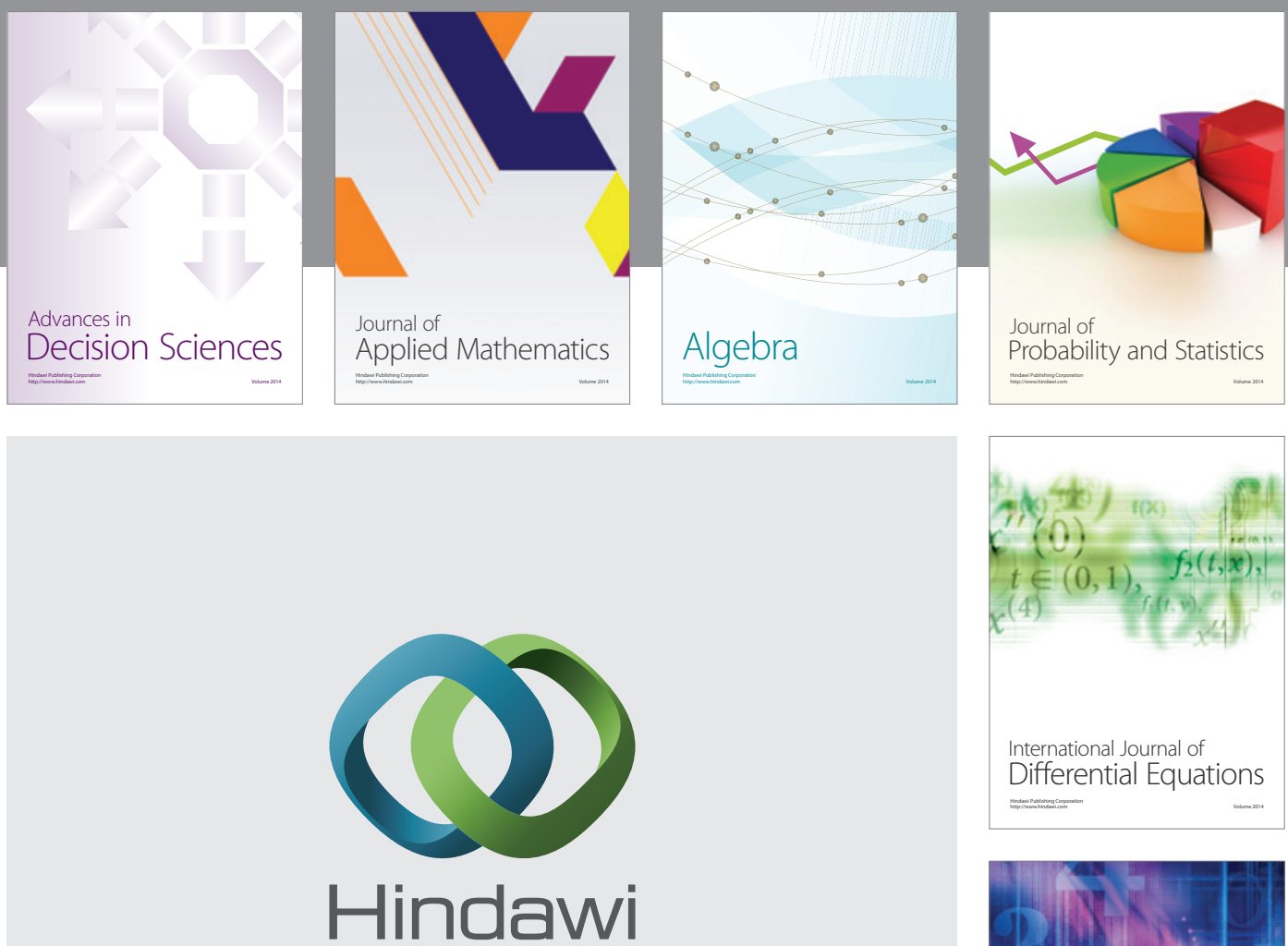

Submit your manuscripts at http://www.hindawi.com
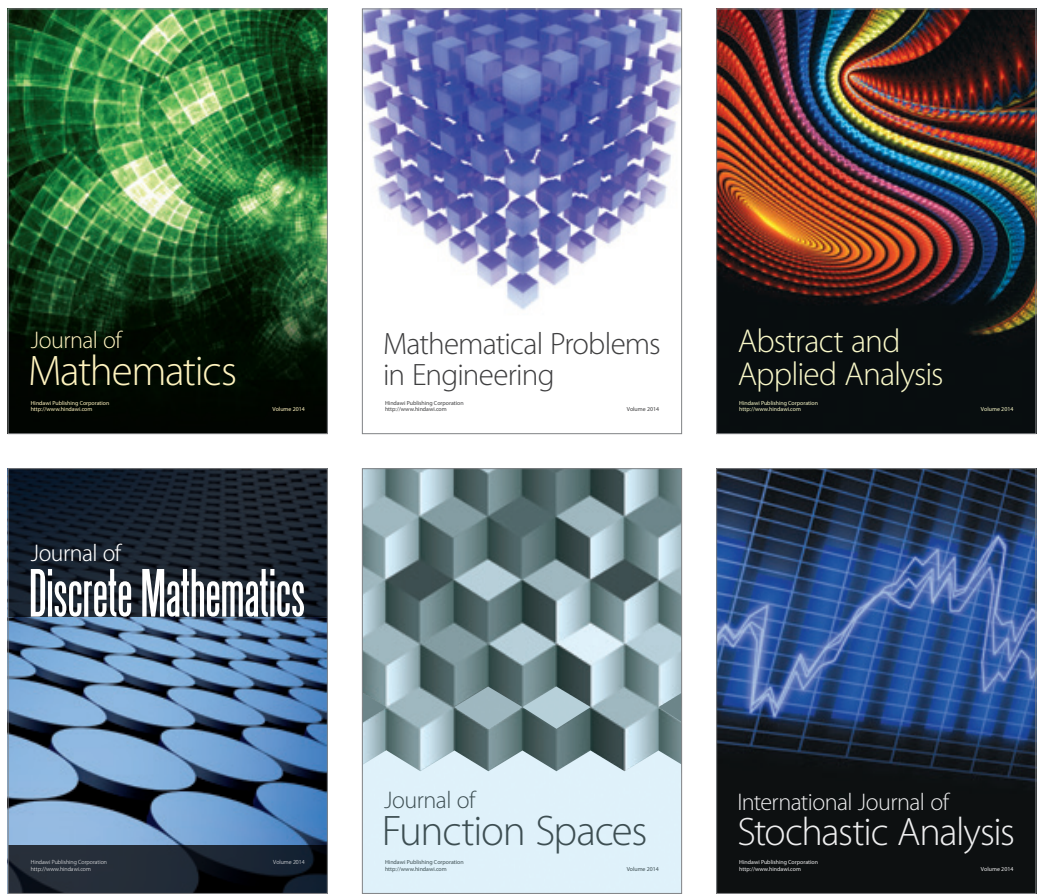

Journal of

Function Spaces

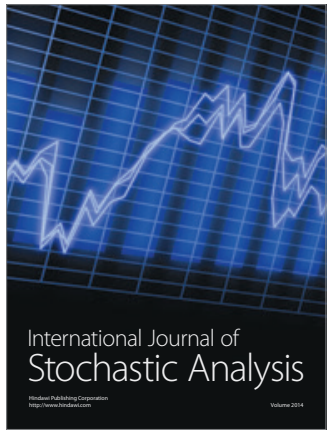

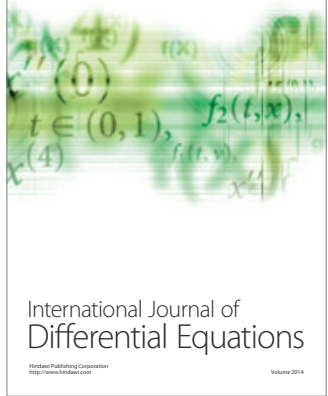
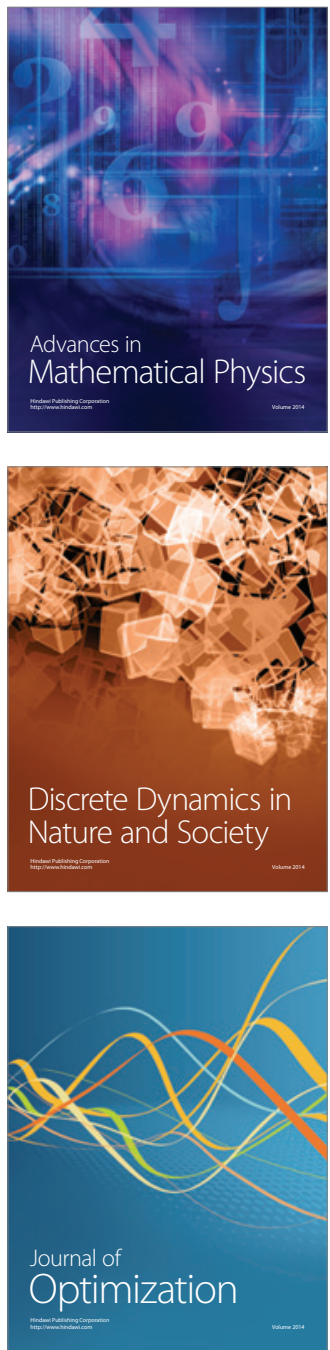